\title{
Potassium permanganate is an excellent alternative to osmium tetroxide in freeze-substitution
}

\author{
Martin Schauflinger ${ }^{1,2}$ (D) $\cdot$ Tim Bergner $^{3} \cdot$ Gregor Neusser $^{4} \cdot$ Christine Kranz $^{4} \cdot$ Clarissa Read $^{3,5}$
}

Accepted: 24 December 2021 / Published online: 5 January 2022

(c) The Author(s) 2022

\begin{abstract}
High-pressure freezing followed by freeze-substitution is a valuable method for ultrastructural analyses of resin-embedded biological samples. The visualization of lipid membranes is one of the most critical aspects of any ultrastructural study and can be especially challenging in high-pressure frozen specimens. Historically, osmium tetroxide has been the preferred fixative and staining agent for lipid-containing structures in freeze-substitution solutions. However, osmium tetroxide is not only a rare and expensive material, but also volatile and toxic. Here, we introduce the use of a combination of potassium permanganate, uranyl acetate, and water in acetone as complementing reagents during the freeze-substitution process. This mix imparts an intense en bloc stain to cellular ultrastructure and membranes, which makes poststaining superfluous and is well suited for block-face imaging. Thus, potassium permanganate can effectively replace osmium tetroxide in the freezesubstitution solution without sacrificing the quality of ultrastructural preservation.
\end{abstract}

Keywords Freeze-substitution $\cdot$ Potassium permanganate $\cdot$ Osmium tetroxide $\cdot$ Uranyl acetate $\cdot$ Membrane stain . Transmission electron microscopy

\section{Introduction}

Rapid cryofixation by high-pressure freezing (HPF) followed by freeze-substitution (FS) results in superior preservation of biological samples compared with conventional electron microscopy (EM) sample preparation protocols that rely on chemical fixation and dehydration of cellular structures at ambient temperatures (Dahl and Staehelin 1989; Shiurba 2001; Zechmann et al. 2007). The HPF-FS method is also compatible with subsequent embedding of the sample in

Martin Schauflinger

martin.schauflinger@staff.uni-marburg.de

1 Institute of Virology, Philipps University Marburg, Hans-Meerwein-Straße 2, 35037 Marburg, Germany

2 Electron Microscopy Core Facility, University of Missouri, 1600 East Rollins Street, Columbia, MO 65211, USA

3 Central Facility for Electron Microscopy, Ulm University, Albert-Einstein-Allee 11, 89081 Ulm, Germany

4 FIB Center UUlm, Institute of Analytical and Bioanalytical Chemistry, Ulm University, Albert-Einstein-Allee 11, 89081 Ulm, Germany

5 Institute of Virology, Ulm University Medical Center, Albert-Einstein-Allee 11, 89081 Ulm, Germany methacrylate or epoxy resins. For these reasons, HPF-FS is the preferred sample preparative method for ambient-temperature transmission electron microscopy (TEM), electron tomography (ET), and block-face imaging using scanning electron microscopy [e.g., serial block-face-scanning electron microscopy (SB-FSEM) and focused ion beam scanning electron microscopy (FIB-SEM)]. During the FS process, the temperature is slowly raised, so that frozen water in the cryoimmobilized sample is gradually replaced by an organic solvent at low temperatures (Shiurba 2001). Fixatives and staining agents can be added to the solvent, which are able to react with cellular components once the temperature is high enough (Humbel and Schwarz 1989).

One of the most important and demanding tasks of biological EM is the visualization of cellular membranes. The preservation and depiction of lipid membranes is critical for the interpretation of cellular ultrastructure. However, depending on the specific biological system and methodology used, it can be challenging to achieve good membrane staining, especially in high-pressure frozen cells (Walther and Ziegler 2002). In many cases, it is necessary to treat sectioned biological specimens with heavy metal salts to achieve sufficient contrast (Reynolds 1963; Ellis 2014). However, as methods that rely on non-sectioned 
specimens - such as tomography and block-face imagingbecome increasingly popular, it is desirable to give the bulk sample an inherently high contrast by increasing the electron density of membranes, i.e., en bloc staining (Jiménez et al. 2009; Webb and Webb 2015; Steyer et al. 2019).

Osmium tetroxide $\left(\mathrm{OsO}_{4}\right)$ is commonly added to FS solutions because of its properties as both a fixative and stain of lipids. $\mathrm{OsO}_{4}$ binds to unsaturated fatty acids, and is regarded as an excellent stain for lipid-containing structures in a range of biological samples. However, $\mathrm{OsO}_{4}$ does not perform well in all cases, and is problematic to handle due to its toxic and volatile nature. Thus, finding osmium alternatives, as well as additives that result in an enhanced membrane staining of freeze-substituted samples, is of great interest. In the past, $\mathrm{OsO}_{4}$ has been used in combination with other chemical agents, such as tannic acid (Giddings 2003; Jiménez et al. 2009), imidazole (Mordhorst et al. 2018, 2019), permanganate (Heath et al. 1985), and even water (Walther and Ziegler 2002; Buser and Walther 2008) to enhance membrane staining.

We explored the use of potassium permanganate $\left(\mathrm{KMnO}_{4}\right)$ as a reagent in FS, based on its ability to provide strong membrane contrast in conventional TEM processing and section poststaining (Luft 1956; Lawn 1960; Kaiser and Schekman 1990; Wright 2000). In this paper, we show that $\mathrm{KMnO}_{4}$ is an excellent en bloc stain for freeze-substituted samples, and provides high inherent electron density to cellular ultrastructure when used in combination with uranyl acetate.

\section{Materials and methods}

Human alveolar basal epithelial cells (A549) or human foreskin fibroblasts (HFF) were grown to near confluency directly on carbon- or gold-coated sapphire disks $(3 \mathrm{~mm}$ in diameter; Engineering Office M. Wohlwend GmbH, Switzerland). Yeast cells [Saccharomyces cerevisiae JD47 (Dohmen et al. 1995)] were grown in suspension medium, concentrated by centrifugation (1000 rpm, $5 \mathrm{~min}$ ), transferred onto carbon-coated and glow-discharged sapphire disks, and let to settle for $1 \mathrm{~min}$ before HPF.

Samples were high-pressure frozen with a Wohlwend HPF device (either Compact 01 or 02; Engineering Office $\mathrm{M}$. Wohlwend $\mathrm{GmbH}$ ) as described previously (Schauflinger et al. 2013; Villinger et al. 2014).

The traditional FS solution consisted of acetone (VWR International $\mathrm{GmbH}$, Darmstadt, Germany) with $0.2 \% \mathrm{OsO}_{4}$ (Merck KGaA, Darmstadt, Germany), 0.2\% uranyl acetate (Merck AG, Darmstadt, Germany), and 5\% water.

The $\mathrm{KMnO}_{4}$ (reagent grade, Merck AG, Darmstadt, Germany) containing FS solutions were prepared by pipetting a small volume of freshly prepared aqueous $\mathrm{KMnO}_{4}$ solution on top of frozen acetone containing uranyl acetate that had been previously frozen solid by immersing cryovials upright in liquid nitrogen. It is important to note that $\mathrm{KMnO}_{4}$ and uranyl acetate will react with each other and precipitate if not mixed at low enough temperatures. The best results (i.e., for mammalian cells) were achieved with FS solutions of acetone containing final concentrations of $0.05 \% \mathrm{KMnO}_{4}$, $0.1-0.2 \%$ uranyl acetate, and $2-5 \%$ water.

Quick FS was carried out in accordance with the superquick freeze-substitution method (SQFS) described previously (McDonald and Webb 2011), with some modifications. Briefly, after HPF, the sapphire disks with the frozen cell monolayers were placed on top of previously frozen FS solution inside a cryovial. The cryovial was then closed tightly and placed into an aluminum block previously cooled to liquid nitrogen temperature. The block was turned sideways and agitated with the help of a rocker operated at $80 \mathrm{rpm}$ until the block turned frost free (after about $1.6 \mathrm{~h}$ ), at which point the samples were transferred to a fridge (ca. $4{ }^{\circ} \mathrm{C}$ ) for $25 \mathrm{~min}$.

Alternatively, automated FS was carried out in the chamber of an AFS2 device (Leica Microsystems GmbH, Wetzlar, Germany) by raising the temperature from $-90{ }^{\circ} \mathrm{C}$ to $4{ }^{\circ} \mathrm{C}$ over a period of $17 \mathrm{~h}$, followed by $30 \mathrm{~min}$ at $4{ }^{\circ} \mathrm{C}$.

The freeze-substituted samples were briefly but thoroughly washed in water-free acetone, embedded in Epon resin/benzyldimethylamine (BDMA) (Embed 812, Science Services) and subsequently polymerized at $60{ }^{\circ} \mathrm{C}$ overnight. Yeast samples were embedded in Epon over $72 \mathrm{~h}$ on a rocker to facilitate complete resin infiltration. Ultrathin silver/grey sections were prepared, mounted on copper grids, and imaged with a JEOL JEM 1400 transmission electron microscope operated at $80 \mathrm{kV}$ or $120 \mathrm{kV}$.

FIB-SEM preparation and imaging of resin-embedded samples was conducted with a Helios Nanolab 600 (Thermo Fisher Scientific, USA), exactly as described previously (Villinger et al. 2015).

\section{Results}

Based on the ability of potassium permanganate $\left(\mathrm{KMnO}_{4}\right)$ to provide strong membrane contrast in conventional EM processing, we explored its use as staining reagent and potential alternative to $\mathrm{OsO}_{4}$ in freeze-substitution (FS). A previous study used $\mathrm{KMnO}_{4}$ for FS of high-pressure frozen yeast, demonstrating the preservation of some ultrastructural features and membranes of HM20 embedded yeast cells (Giddings 2003). In our tests, the incorporation of $\mathrm{KMnO}_{4}$ to the FS solution without the addition of other reagents did not provide consistent results nor sufficient staining in cultured mammalian cells (Fig. 1). Usually, the addition of $\mathrm{KMnO}_{4}$ to acetone resulted in a relatively weak staining of the 
nuclear lamina, cytoskeletal elements, and the mitochondrial matrix, while ribosomes were clearly visible. Occasionally, we observed a weak staining of some membranes and an increased electron density of the ER lumen (Fig. 1a). Most of the time, the contrast was extremely weak and membranes were either not or only barely visible, even with extended FS times or the addition of water (Fig. 1b, c). Furthermore, the use of $\mathrm{KMnO}_{4}$ under these conditions could lead to the formation of artifacts such as electron dense deposits (Fig. 1a) or white spots (Fig. 1c). Similar problems were observed when the concentration of $\mathrm{KMnO}_{4}$ was too high, which is why we used working concentrations of well below $0.1 \%$ $(\mathrm{w} / \mathrm{v}) \mathrm{KMnO}_{4}$ in the following experiments.
Since $\mathrm{KMnO}_{4}$ by itself was not sufficient as a fixative or staining agent, we explored the supplementation of additional chemical agents to the FS solution. We found that the combination of $\mathrm{KMnO}_{4}$ and uranyl acetate returned promising results in freeze-substituted mammalian cells (Fig. 2). To circumvent the precipitation of $\mathrm{KMnO}_{4}$ and uranyl acetate, which happened upon mixing both agents together at room temperature, we exploited the fact that $\mathrm{KMnO}_{4}$ and uranyl acetate did not react with each other at low temperatures. Thus, one part of the solution was frozen first (i.e., uranyl acetate in acetone), and only then was the $\mathrm{KMnO}_{4}$ solution added. Low concentrations of $\mathrm{KMnO}_{4}$ were sufficient to produce very strong staining when uranyl acetate was included
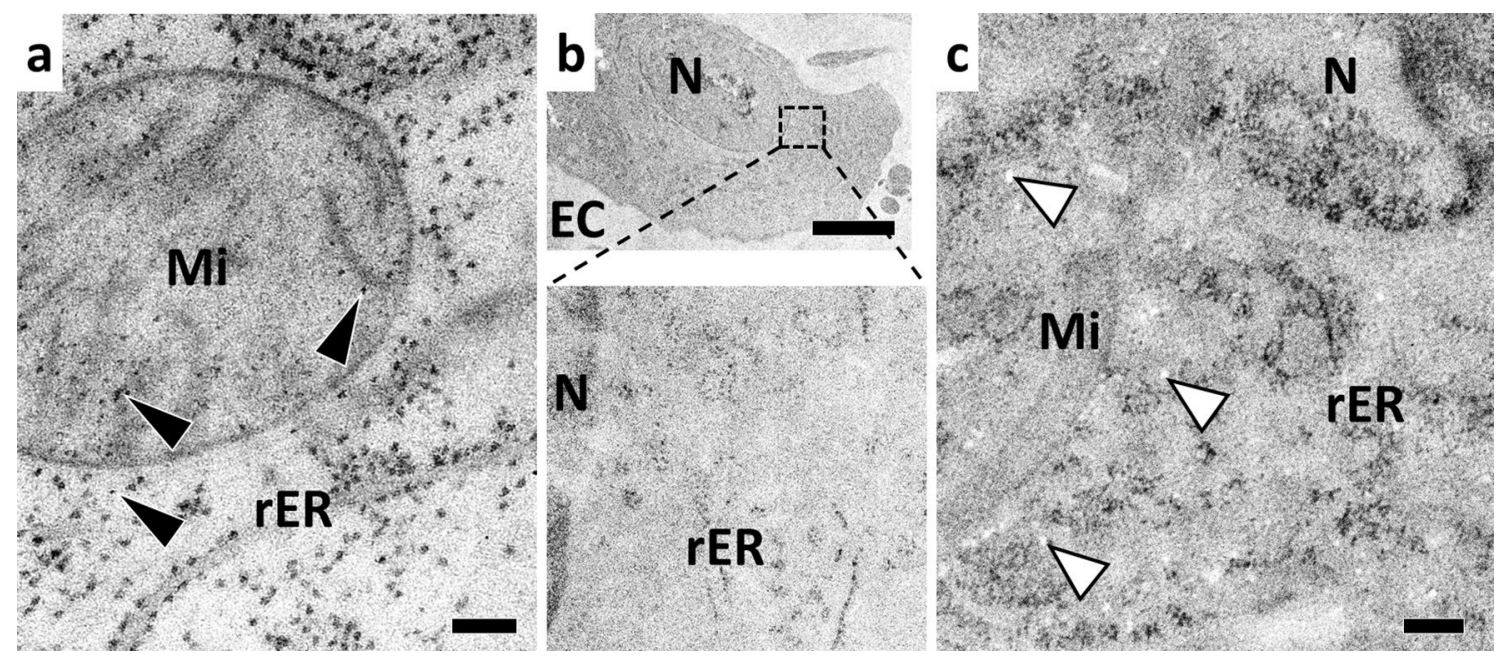

Fig. $1 \mathrm{KMnO}_{4}$ alone is not a potent membrane stain in freeze-substituted mammalian cells. A549 cells were high-pressure frozen, freezesubstituted, and embedded in Epon, and sections were observed using TEM. a A549 cells super-quick freeze-substituted (SQFS) with $0.2 \%$ $\mathrm{KMnO}_{4}$. b SQFS of A549 with $0.05 \% \mathrm{KMnO}_{4}$ and $2 \%$ water. c HFF freeze-substituted over a period of $17 \mathrm{~h}$ in acetone containing $0.05 \%$ $\mathrm{KMnO}_{4}$ and 5\% water. Black arrows depict artifactual electron dense spots, white arrows depict artifactual white spots. Bars: $100 \mathrm{~nm}(\mathbf{a})$, $5 \mu \mathrm{m}(\mathbf{b}), 1 \mu \mathrm{m}(\mathbf{c}) . M i$ mitochondrion, $r E R$ rough endoplasmic reticulum, $N$ nucleus, $E C$ extracellular space

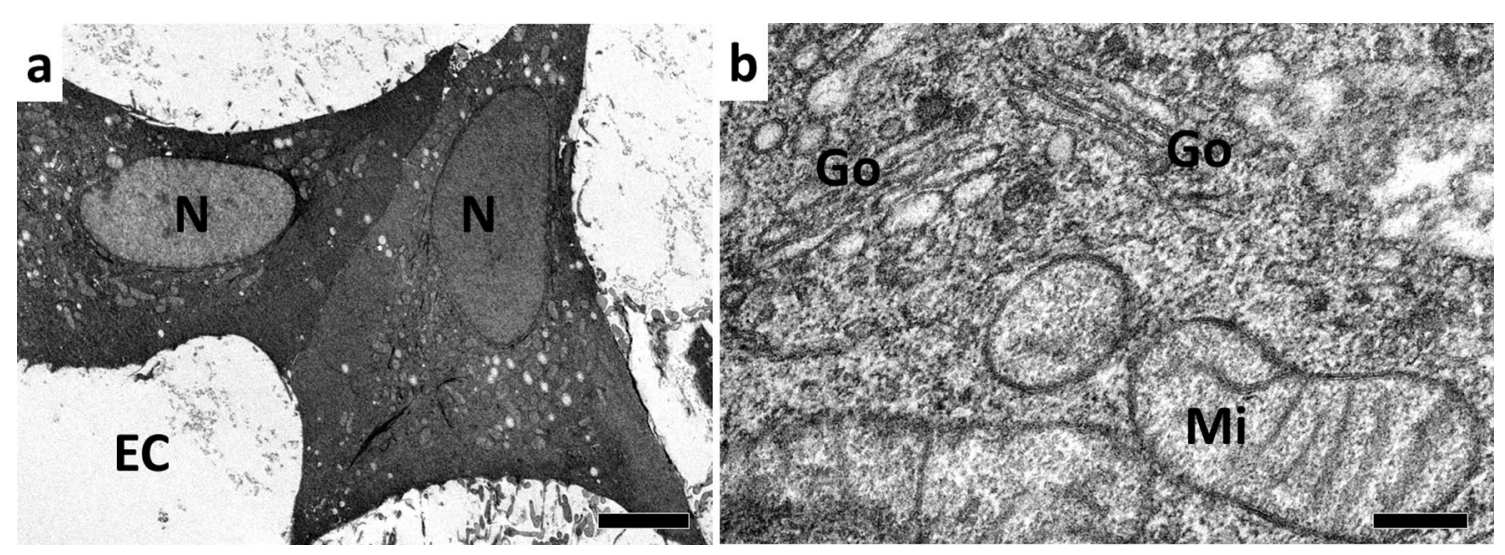

Fig. $2 \mathrm{KMnO}_{4}$ in combination with uranyl acetate results in very intense staining of cellular ultrastructure. A549 cells were high-pressure frozen followed by SQFS with $0.05 \% \mathrm{KMnO}_{4}$ and $0.2 \%$ uranyl acetate, embedded in Epon, and sections were observed using TEM. a Overview, b detail. Bars: $5 \mu \mathrm{m}(\mathbf{a}), 200 \mathrm{~nm}(\mathbf{b})$. ( $N$ nucleus, $E C$ extracellular space, Go Golgi apparatus, $M i$ mitochondrion) 
in the FS solution, and the membranes showed very clearly the railroad track morphology of the lipid bilayer (Fig. 2b). However, the cytoplasm appeared relatively dark, which made it difficult to distinguish individual features, such as organelles or cytoskeletal structures.

Interestingly, the addition of a small amount of water to the FS solution resulted in a much less intense staining of the cytoplasm, seemingly without affecting the strong staining of other ultrastructural features, such as the lipid bilayers and microtubules (Fig. 3). This led to increased visibility of individual organelles and cytoskeletal elements, which allowed detailed analysis of the cellular ultrastructure. The combination of $\mathrm{KMnO}_{4}$, uranyl acetate, and water described here worked equally well with both the more conventional FS times ( $>16 \mathrm{~h})$ and the very short FS schedules described more recently as super-quick freeze-substitution (SQFS) (McDonald and Webb 2011). To achieve sufficient staining, it appeared beneficial to incubate the samples at a temperature slightly above $0{ }^{\circ} \mathrm{C}$ for at least a few minutes at the end of each FS run. On the other hand, it was critical not to let the sample reach room temperature, because this usually resulted in artifacts such as overstaining and degradation of the fine cellular ultrastructure.

For comparison, we simultaneously processed highpressure frozen HFF cells in a standard FS solution containing $\mathrm{OsO}_{4}$. The results achieved with the FS mixture of $0.05 \% \mathrm{KMnO}_{4}, 0.2 \%$ uranyl acetate, and $5 \%$ water in acetone (Fig. 3, left panel) were almost indistinguishable from the FS solution used routinely in the Ulm laboratory, which consists of $0.2 \% \mathrm{OsO}_{4}, 0.2 \%$ uranyl acetate, and $5 \%$ water
Fig. 3 FS with a combination of $\mathrm{KMnO}_{4}$, uranyl acetate, and water (left panel) results in excellent preservation and staining of the cellular ultrastructure, similar to a standard FS solution that contains $\mathrm{OsO}_{4}$, uranyl acetate, and water (right panel). HFF were high-pressure frozen and freeze-substituted over $17 \mathrm{~h}$ in acetone containing $0.2 \%$ uranyl acetate, $5 \%$ water, and additionally either $0.05 \%$ $\mathrm{KMnO}_{4}$ (left panel) or $0.2 \%$ $\mathrm{OsO}_{4}$ (right panel). The cells were then embedded in Epon, and ultrathin sections were observed on TEM at $80 \mathrm{kV}$. a Low magnification overview, b perinuclear region, $\mathbf{c}$ higher magnification of the centrosomal region showing details of lipid bilayers (white arrows) and microtubules (black arrows).

Bars: $5 \mu \mathrm{m}(\mathbf{a}), 1 \mu \mathrm{m}(\mathbf{b})$, $250 \mathrm{~nm}$ (c). Mi mitochondrion, $N$ nucleus, $E C$ extracellular space, Go Golgi apparatus
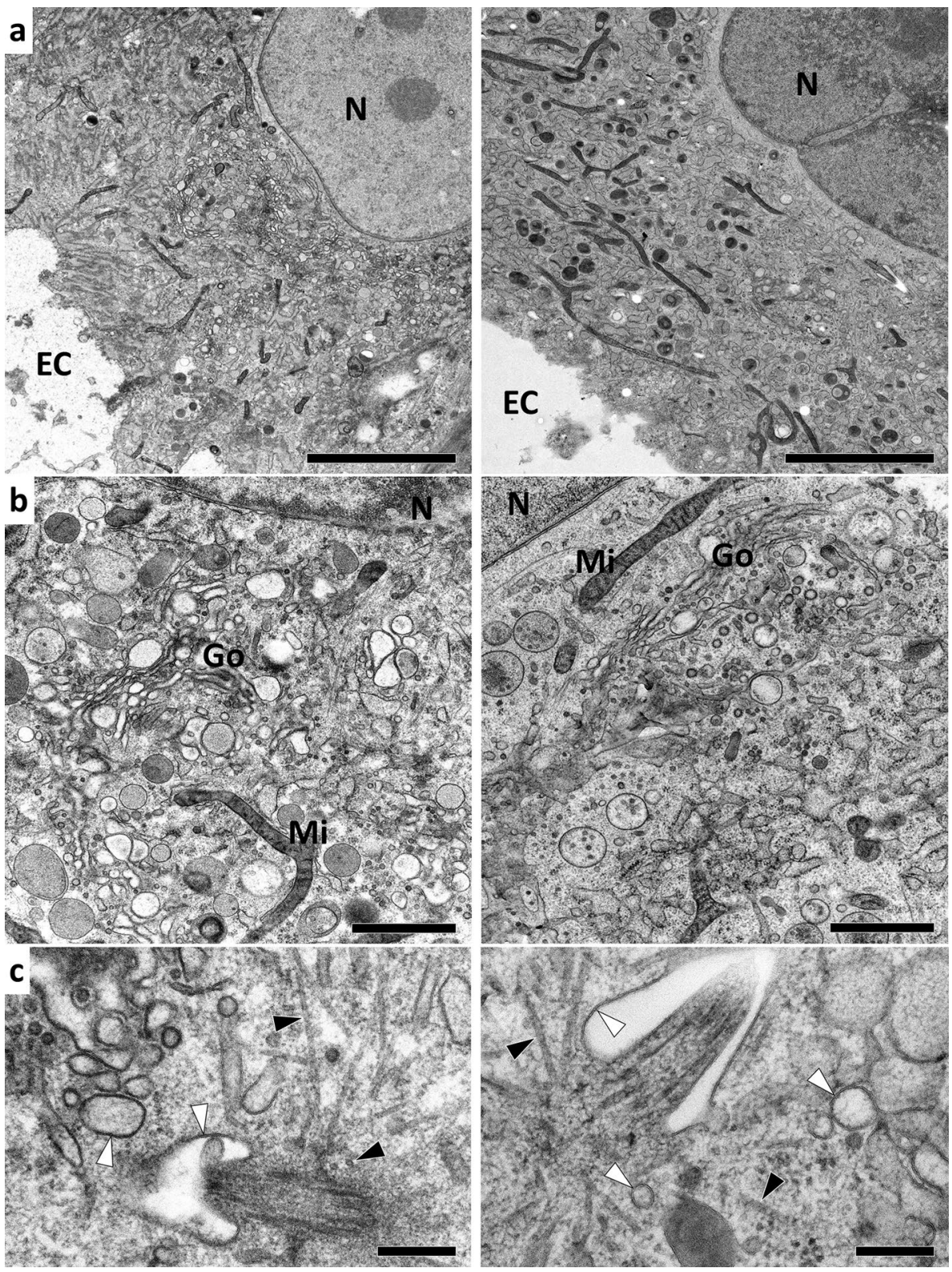
in acetone (Fig. 3, right panel). In comparison, the $\mathrm{KMnO}_{4}$ treated cells exhibited a stronger staining intensity, which was especially noticeable on the TEM viewing screen, where it was possible to easily distinguish features of the $\mathrm{KMnO}_{4}$ treated samples by eye, while $\mathrm{OsO}_{4}$ treatment resulted in a relatively weak contrast.

The interaction between $\mathrm{KMnO}_{4}$ and uranyl acetate appeared to be responsible for the excellent staining of membranes, which can be demonstrated by observing sections after using uranyl acetate as the sole ingredient with acetone in the FS solution (Fig. 4). While uranyl acetate stained structures rich in nucleic acids, such as ribosomes and cell nuclei, as well as cytoskeletal structures, it was not suitable as a membrane stain. Since neither $\mathrm{KMnO}_{4}$ nor uranyl acetate alone stained membranes, a complementary action of both agents likely caused the well-defined membrane staining observed in the presence of $\mathrm{KMnO}_{4}$ and uranyl acetate together.
Since $\mathrm{KMnO}_{4}$ has historically been used especially for TEM preparation of yeast, we also explored its applicability on high-pressure frozen samples of S. cerevisiae (Fig. 5). We achieved decent staining of the cellular components when using a combination of $0.05 \% \mathrm{KMnO}_{4}, 0.2 \%$ uranyl acetate, and $5 \%$ water, and could observe well-defined membranes as well as microtubules. These results were comparable to FS with $\mathrm{OsO}_{4}$ (not shown), and indicate that FS with a combination of $\mathrm{KMnO}_{4}$, uranyl acetate, and water could likely be adapted for ultrastructural analyses of various (micro-) biological samples.

Finally, we wanted to determine if FS with a combination of $\mathrm{KMnO}_{4}$, uranyl acetate, and water was suited for use in block-face imaging, especially for focused ion beam scanning electron microscopy (FIB-SEM). This method has become quite popular with biologists, but as there is no possibility of post-section staining, it requires sufficient en bloc stain. Image formation in SEM differs from TEM.
Fig. 4 Uranyl acetate alone is not a sufficient membrane staining agent. A549 cells were high-pressure frozen, SQFS with acetone containing $0.2 \%$ uranyl acetate and $2 \%$ water, embedded in Epon, and sections were observed using TEM. a Overview, b detail. Bars: $2 \mu \mathrm{m}$ (a), $200 \mathrm{~nm}$ (b). $N$ nucleus, $E C$ extracellular space, $r E R$ rough endoplasmic reticulum, $M i$ mitochondrion, $M t$ microtubules
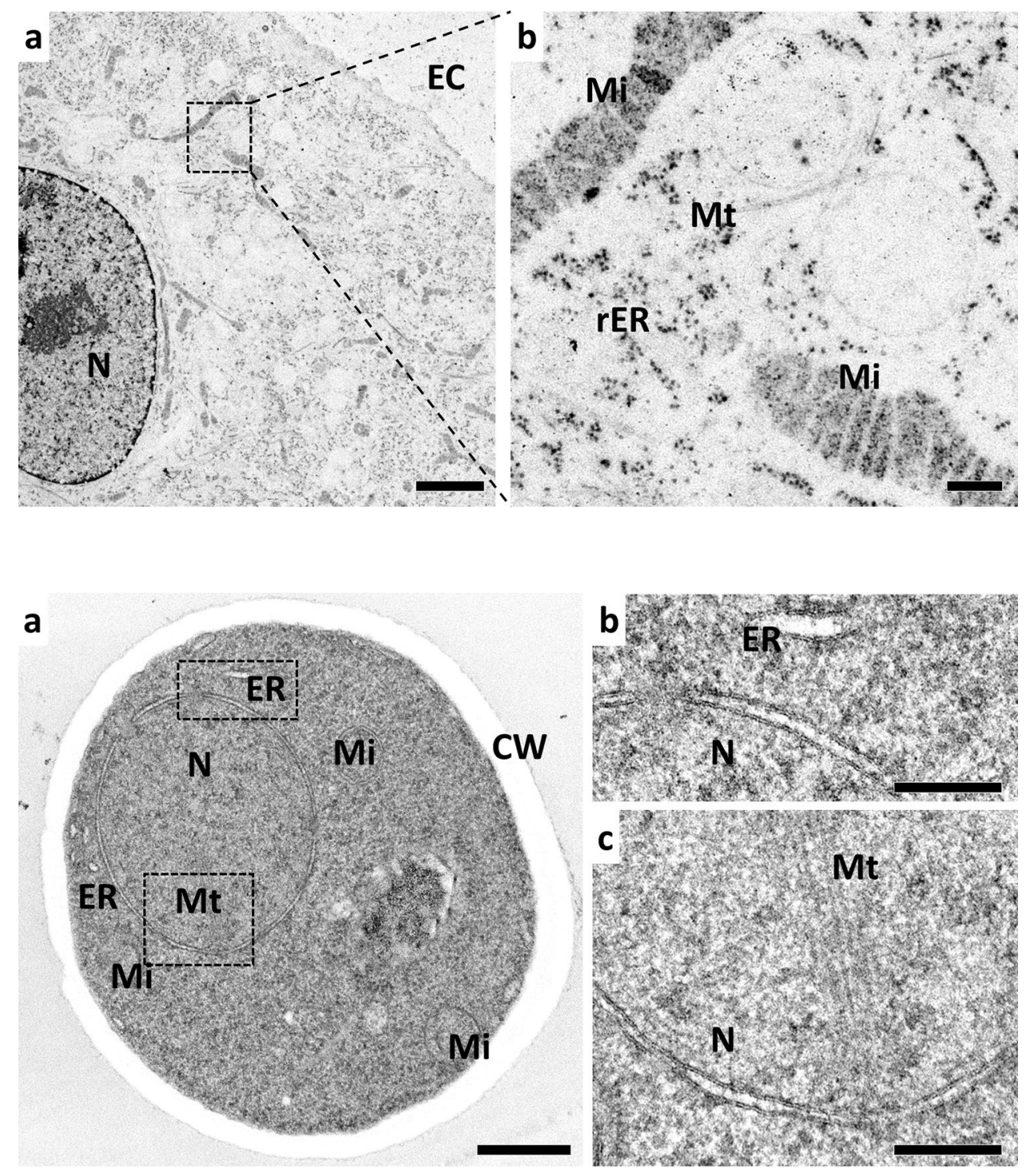

Fig. 5 FS with $\mathrm{KMnO}_{4}$, uranyl acetate, and water provides good visualization of membranes and microtubules of a yeast cell. A suspension of S. cerevisiae was high-pressure frozen, freeze-substituted over a period of $17 \mathrm{~h}$ in acetone containing $0.05 \% \mathrm{KMnO} 4,0.2 \%$ uranyl acetate, and 5\% water. and embedded in Epon, and sections were observed using TEM at $120 \mathrm{kV}$. Bars: $500 \mathrm{~nm}(\mathbf{a})$, $200 \mathrm{~nm}(\mathbf{b}, \mathbf{c}) . E R$ endoplasmic reticulum, $N$ nucleus, $M i$ mitochondrion, $C W$ cell wall, $\mathrm{Mt}$ microtubules of the spindle pole body 
While the contrast in TEM is created by the transmission of electrons through a thin sample, the SEM image is formed by detecting secondary or backscattered electrons originating from the block face. Thus, the conductivity of the investigated block face needs to be high enough to prevent sample charging during SEM acquisition. Remarkably, in high-pressure frozen mammalian cells, FS with a combination of $\mathrm{KMnO}_{4}$, uranyl acetate, and water produced strong en bloc staining, which was suitable for FIB-SEM analyses of resin-embedded samples (Fig. 6). The cells were sufficiently stained to be recognizable under the resin surface at $15 \mathrm{kV}$ acceleration voltage, which is important for localizing cells of interest (Fig. 6a). Slice and view imaging at $5 \mathrm{kV}$, and detection of the secondary electron signal with the through-the-lens detector resulted in images with TEM-like resolution (Fig. 6b-d), comparable to the quality achieved with FS solutions containing $\mathrm{OsO}_{4}$, uranyl acetate, and water (Villinger et al. 2012).

\section{Discussion}

This study was born out of the necessity to find a suitable replacement for $\mathrm{OsO}_{4}$ in the laboratory of the EM Core Facility of the University of Missouri. In this paper, we show that $\mathrm{KMnO}_{4}$ in combination with uranyl acetate in the FS solution can effectively replace $\mathrm{OsO}_{4}$ as a fixative and staining agent in different cultured cells. The addition of water to the FS solution lowered the overall staining intensity and thus enhanced the appearance of the cellular ultrastructure. In general, the staining of biological features appeared to be stronger when $\mathrm{KMnO}_{4}$ instead of $\mathrm{OsO}_{4}$ was used, which for most purposes should render poststaining of sections unnecessary.

Aqueous $\mathrm{KMnO}_{4}$ was one of the first fixatives to be used for EM preparations (Luft 1956). It still has its place as postfixative, with or without additional aldehyde fixation, because of its good membrane preserving properties, especially for the preparation of yeast cells (Wright 2000; Heiman and Walter 2000). However, permanganate was not seen as a general alternative to $\mathrm{OsO}_{4}$, although membranes were preserved and contrasted well. Aqueous permanganate solutions did not preserve cytoskeletal and other nonlipid structures; it altered the morphology of some organelles and degraded fine ultrastructure when employed as fixative at ambient temperature (Luft 1956; Frankl et al. 2015). These detrimental effects are probably from the strong oxidizing property of permanganate that affects nonmembranous structures (Hopwood 1969; Hayat 1993; Wright 2000). There could be a number of reasons for the prevention or mitigation of detrimental effects by $\mathrm{KMnO}_{4}$ in FS, for instance, the reduced reactivity of permanganate at low temperatures and low concentrations of water, as well as the presence of uranyl acetate, which seems to serve as a reaction partner of permanganate. The latter could be responsible for a further decrease in the concentration of the already relatively low amounts of $\mathrm{KMnO}_{4}$ in the FS solution, thus limiting the chance of detrimental reactions with the sample material. Consistently, we and others have noticed harmful effects of $\mathrm{KMnO}_{4}$ exacerbated by increased working concentrations of $\mathrm{KMnO}_{4}$, as well as by higher temperatures at the end of the FS run (Giddings 2003).

Based on its ability to provide strong membrane contrast in conventional EM processing and its use for section poststaining, $\mathrm{KMnO}_{4}$ had previously been explored as reagent in
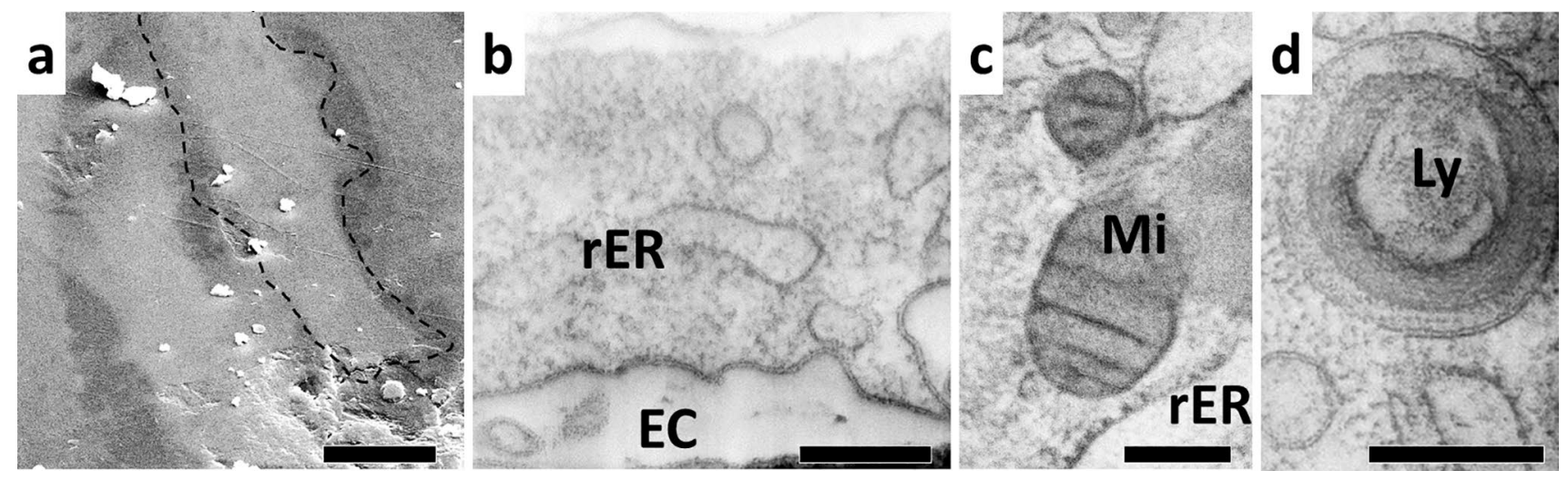

Fig. 6 FS with $\mathrm{KMnO}_{4}$ and uranyl acetate is suitable for FIB-SEM analyses. HFF were high-pressure frozen, freeze-substituted over a period of $17 \mathrm{~h}$ in acetone containing $0.05 \% \mathrm{KMnO}_{4}, 0.2 \%$ uranyl acetate, and 5\% water, and embedded in Epon. The resin-embedded sample was mounted on a stub, and then a block face was created and imaged by FIB-SEM. a View onto the embedded cells at $15 \mathrm{kV}$ accel- eration voltage before milling into the outlined cell. b-d Views from the block face after milling, taken by detection of secondary electrons at $5 \mathrm{kV}$ (inverted contrast). Bars: $15 \mu \mathrm{m}(\mathbf{a}), 250 \mathrm{~nm}(\mathbf{b}-\mathbf{d}) . r E R$ rough endoplasmic reticulum, $E C$ extracellular space, $M i$ mitochondrion, Ly lysosome 
FS of high-pressure frozen samples, with somewhat underwhelming results (Giddings 2003; Hess 2003). One study recommended a systematic analysis of the optimum concentration, temperature, and time parameters for FS with $\mathrm{KMnO}_{4}$ in acetone (Giddings 2003). However, as we demonstrate in the present paper, the true potential of $\mathrm{KMnO}_{4}$ as a staining agent appears to be unlocked by its interaction with uranyl acetate. Uranyl acetate has been in use for a long time as both a fixative and heavy metal stain in TEM analyses of biological samples. However, uranyl acetate alone is not a sufficient membrane staining agent, and evidence suggests that the previously reported effect of uranyl acetate on the marked improvement in the preservation of cell membranes is due to a fixating rather than a contrasting action (Silva et al. 1968, 1971). On the other hand, $\mathrm{KMnO}_{4}$ has been noted as an excellent reagent for revealing and studying membrane structures within the cell, but was considered to act as a stain rather than a true fixative (Bradbury and Meek 1960; Wright 2000). Thus, the excellent membrane staining property of the $\mathrm{KMnO}_{4}$ in combination with uranyl acetate in the FS solution reported here is most likely mediated by the complementary reaction of $\mathrm{KMnO}_{4}$ with (membranebound) uranyl acetate. The fact that $\mathrm{KMnO}_{4}$ and uranyl acetate precipitate when mixed together at ambient temperature is evidence for an interaction between these reagents.

Initially, the addition of up to $10 \%$ of water to the FS solution was introduced as a way to improve membrane staining in combination with $\mathrm{OsO}_{4}$, even though the precise mechanism of this action is not entirely clear (Walther and Ziegler 2002; Buser and Walther 2008). Water-free FS with $\mathrm{KMnO}_{4}$ and uranyl acetate imparts intense staining to the membrane bilayers, but can also darken the cytoplasm considerably. Addition of water to this $\mathrm{KMnO}_{4}$ and uranyl acetate containing FS solution causes a marked decrease in the staining intensity of the cytoplasm without affecting the membrane staining, which allows an easier differentiation of individual structures and organelles. Although the processes underlying the observed effect are not clarified, it appears as if the added water affects the interactions between $\mathrm{KMnO}_{4}$, uranyl acetate, and/or the sample materials. We could observe good results for mammalian cells upon adding $2-5 \%$ of water to the FS solutions containing $\mathrm{KMnO}_{4}$ and uranyl acetate. The optimal balance of these ingredients in FS solutions will likely depend on the respective biological sample and question of interest, and thus may require more empirical testing and optimization for different specimens and methods.

We could successfully demonstrate the high resolution block-face imaging of $\mathrm{KMnO}_{4}$ stained FIB-SEM samples by detecting the secondary electron signal, in line with our previous work for $\mathrm{OsO}_{4}$ treated samples (Villinger et al. 2012). One advantage of secondary electron block-face images are that the measurable secondary electrons are produced close to the surface, which is beneficial in achieving a high resolution. Backscattered electron imaging, on the other hand, usually provides a higher contrast at the expense of resolution (Winter et al. 2009). One would expect a lower backscattered electron signal when osmium (atomic weight 190 ) is exchanged with manganese (atomic weight 55), with a considerable amount of signal being contributed by scattering at the uranium (atomic weight 238). Thus we recommend using secondary electron detection for block-face imaging of $\mathrm{KMnO}_{4}$ stained samples. Taken together, our data demonstrates that $\mathrm{OsO}_{4}$ in the FS solution can be replaced by a low concentration of $\mathrm{KMnO}_{4}$ for ultrastructural investigations of high-pressure frozen, freeze-substituted, resin-embedded cells. A replacement of $\mathrm{OsO}_{4}$ by $\mathrm{KMnO}_{4}$ has several advantages, while the achieved ultrastructural preservation and staining is at least equivalent. The use of $\mathrm{KMnO}_{4}$ contributes to workplace and environmental safety, since $\mathrm{OsO}_{4}$ crystals and solutions are volatile, toxic, and must be handled and disposed of very carefully. Furthermore, $\mathrm{KMnO}_{4}$ is abundant and much cheaper compared with the relatively rare and expensive $\mathrm{OsO}_{4}$. Finally, $\mathrm{KMnO}_{4}$ can serve as an alternative in cases where $\mathrm{OsO}_{4}$ does not perform well.

Acknowledgements We thank Senthil R. Kumar (Department of Veterinary Medicine \& Surgery, University of Missouri) for providing A549 cells, and N. Johnsson and Ch. Pfeiffer (Institute of Molecular Genetics and Cell Biology, Ulm University) for providing yeast cells.

Author contributions M.S. conceived the study, performed experiments, and wrote the paper; T.B. performed TEM experiments; G.N. and C.K. performed FIB-SEM analysis; C.R. supervised TEM and FIBSEM experiments, and critically reviewed the manuscript; all authors approved of the final version of the manuscript.

Funding Open Access funding enabled and organized by Projekt DEAL. This work was supported by the University of Missouri Office of Research, and by the Deutsche Forschungsgemeinschaft (DFG, German Research Foundation) through the Collaborative Research Centre 1279 (\#316249678) and Collaborative Research Centre 1021 (\#197785619). The Focused Ion Beam Center UUlm was supported by Thermo Fisher (formerly FEI), the DFG (INST40/385-F1UG), and the Struktur- und Innovationsfonds Baden-Württemberg.

Data availability The data generated during the current study are available from the corresponding author on reasonable request.

\section{Declarations}

Conflict of interest The authors declare no conflicts of interest.

Open Access This article is licensed under a Creative Commons Attribution 4.0 International License, which permits use, sharing, adaptation, distribution and reproduction in any medium or format, as long as you give appropriate credit to the original author(s) and the source, provide a link to the Creative Commons licence, and indicate if changes were made. The images or other third party material in this article are included in the article's Creative Commons licence, unless indicated otherwise in a credit line to the material. If material is not included in the article's Creative Commons licence and your intended use is not permitted by statutory regulation or exceeds the permitted use, you will 
need to obtain permission directly from the copyright holder. To view a copy of this licence, visit http://creativecommons.org/licenses/by/4.0/.

\section{References}

Bradbury S, Meek GA (1960) A study of potassium permanganate 'fixation' for electron microscopy. J Cell Sci 55:241-250. https:// doi.org/10.1242/jcs.s3-101.55.241

Buser C, Walther P (2008) Freeze-substitution: the addition of water to polar solvents enhances the retention of structure and acts at temperatures around $-60^{\circ} \mathrm{C}$. J Microsc 230:268-277. https://doi. org/10.1111/j.1365-2818.2008.01984.x

Dahl R, Staehelin LA (1989) High-pressure freezing for the preservation of biological structure: theory and practice. J Electron Microsc Tech 13:165-174. https://doi.org/10.1002/jemt.10601 30305

Dohmen RJ, Stappen R, McGrath JP et al (1995) An essential yeast gene encoding a homolog of ubiquitin-activating enzyme. J Biol Chem 270:18099-21810. https://doi.org/10.1074/jbc.270.30. 18099

Ellis EA (2014) Staining sectioned biological specimens for transmission electron microscopy: conventional and En bloc stains. In: Kuo J (ed) Electron Microscopy. Humana Press, Totowa. https:// doi.org/10.1007/978-1-62703-776-1_4

Frankl A, Mari M, Reggiori F (2015) Electron microscopy for ultrastructural analysis and protein localization in Saccharomyces cerevisiae. Microbial Cell 2:412-428. https://doi.org/10.15698/ mic2015.11.237

Giddings TH (2003) Freeze-substitution protocols for improved visualization of membranes in high-pressure frozen samples. J Microsc 212:53-61. https://doi.org/10.1046/j.1365-2818.2002.01064.x

Hayat MA (1993) Stains and cytochemical methods, 1st edn. Springer, US

Heath IB, Rethoret K, Arsenault AL, Ottensmeyer FP (1985) Improved preservation of the form and contents of wall vesicles and the golgi apparatus in freeze substituted hyphae of Saprolegnia. Protoplasma 128:81-93. https://doi.org/10.1007/BF01276331

Heiman MG, Walter P (2000) Prm1p, a pheromone-regulated multispanning membrane protein, facilitates plasma membrane fusion during yeast mating. J Cell Biol 151:719-730. https://doi.org/10. 1083/jcb.151.3.719

Hess MW (2003) Of plants and other pets: practical aspects of freezesubstitution and resin embedding. J Microsc 212:44-52. https:// doi.org/10.1046/j.1365-2818.2003.01237.x

Hopwood D (1969) Fixation of proteins by osmium tetroxide potassium dichromate and potassium permanganate: model experiments with bovine serum albumin and bovine $\gamma$-globulin. Histochemie 18:250-260. https://doi.org/10.1007/BF00306171

Humbel BM, Schwarz H (1989) Freeze-substitution for immunocytochemistry. In: Verkleij AJ, Leunissen JLM (eds) Immunogold labeling in cell biology. CRC Press, Boca Raton, pp 115-134

Jiménez N, Vocking K, van Donselaar EG et al (2009) Tannic acidmediated osmium impregnation after freeze-substitution: a strategy to enhance membrane contrast for electron tomography. $\mathrm{J}$ Struct Biol 166:103-106. https://doi.org/10.1016/j.jsb.2008.12. 009

Kaiser CA, Schekman R (1990) Distinct sets of SEC genes govern transport vesicle formation and fusion early in the secretory pathway. Cell 61:723-733. https://doi.org/10.1016/0092-8674(90) 90483-U

Lawn AM (1960) The use of potassium permanganate as an electrondense stain for sections of tissue embedded in epoxy resin. $\mathrm{J}$
Biophys Biochem Cytol 7:197-198. https://doi.org/10.1083/ jcb.7.1.197

Luft JH (1956) Permanganate-a new fixative for electron microscopy. J Cell Biol 2:799-802. https://doi.org/10.1083/jcb.2.6.799

McDonald KL, Webb RI (2011) Freeze substitution in 3 hours or less. J Microsc 243:227-233. https://doi.org/10.1111/j.1365-2818.2011. 03526.x

Mordhorst BR, Murphy SL, Schauflinger M et al (2018) Porcine fetalderived fibroblasts alter gene expression and mitochondria to compensate for hypoxic stress during culture. Cell Reprogram 20:225-235. https://doi.org/10.1089/cell.2018.0008

Mordhorst BR, Kerns KC, Schauflinger M et al (2019) Pharmacologic treatment with CPI-613 and PS48 decreases mitochondrial membrane potential and increases quantity of autolysosomes in porcine fibroblasts. Sci Rep 9:9417. https://doi.org/10.1038/ s41598-019-45850-4

Reynolds ES (1963) The use of lead citrate at high $\mathrm{pH}$ as an electronopaque stain in electron microscopy. J Cell Biol 17:208-212. https://doi.org/10.1083/jcb.17.1.208

Schauflinger M, Villinger C, Mertens T et al (2013) Analysis of human cytomegalovirus secondary envelopment by advanced electron microscopy: HCMV morphogenesis. Cell Microbiol 15:305-314. https://doi.org/10.1111/cmi.12077

Shiurba R (2001) Freeze-substitution: origins and applications. Int Rev Cytol 206:45-96. https://doi.org/10.1016/s0074-7696(01)06019-3

Silva MT, Carvalho Guerra F, Magalhães MM (1968) The fixative action of uranyl acetate in electron microscopy. Experientia 24:1074-1074. https://doi.org/10.1007/BF02138757

Silva MT, Santos Mota JM, Melo JVC, Carvalho Guerra F (1971) Uranyl salts as fixatives for electron microscopy: study of the membrane ultrastructure and phospholipid loss in bacilli. Biochim Biophys Acta Biomembr 223:513-520. https://doi.org/10.1016/ 0005-2736(71)90240-9

Steyer AM, Ruhwedel T, Möbius W (2019) Biological sample preparation by high-pressure freezing, microwave-assisted contrast enhancement, and minimal resin embedding for volume imaging. J vis Exp. https://doi.org/10.3791/59156

Villinger C, Gregorius H, Kranz C et al (2012) FIB/SEM tomography with TEM-like resolution for 3D imaging of high-pressure frozen cells. Histochem Cell Biol 138:549-556. https://doi.org/10.1007/ s00418-012-1020-6

Villinger C, Schauflinger M, Gregorius H et al (2014) Three-dimensional imaging of adherent cells using FIB/SEM and STEM. In: Kuo J (ed) Electron Microscopy. Humana Press, Totowa, pp 617638. https://doi.org/10.1007/978-1-62703-776-1_27

Villinger C, Neusser G, Kranz C et al (2015) 3D Analysis of HCMV induced-nuclear membrane structures by FIB/SEM tomography: insight into an unprecedented membrane morphology. Viruses 7:5686-5704. https://doi.org/10.3390/v7112900

Walther P, Ziegler A (2002) Freeze substitution of high-pressure frozen samples: the visibility of biological membranes is improved when the substitution medium contains water. J Microsc 208:3-10. https://doi.org/10.1046/j.1365-2818.2002.01064.x

Webb R, Webb R (2015) Quick freeze substitution processing of biological samples for serial block-face scanning electron microscopy. Microsc Microanal 21:1115-1116. https://doi.org/10.1017/ S1431927615006364

Winter DMD, Schneijdenberg C, Lebbink M et al (2009) Tomography of insulating biological and geological materials using focused ion beam (FIB) sectioning and low-kV BSE imaging. J Microsc 233:372-383. https://doi.org/10.1111/j.1365-2818.2009.03139.x

Wright R (2000) Transmission electron microscopy of yeast. Microsc Res Tech 51:469-510. https://doi.org/10.1002/1097-0029(20001 215)51:6\%3c496::AID-JEMT2\%3e3.0.CO;2-9 
Zechmann B, Müller M, Zellnig G (2007) Membrane associated qualitative differences in cell ultrastructure of chemically and high pressure cryofixed plant cells. J Struct Biol 158:370-377. https:// doi.org/10.1016/j.jsb.2006.12.003
Publisher's Note Springer Nature remains neutral with regard to jurisdictional claims in published maps and institutional affiliations. 